\title{
Etude des possibilités de mesure des transactinides par spectrométrie de photons de faible énergie
}

\author{
J. MOREL, M.-C. LEPY, B. CHAUVENET* \\ (Manuscrit reçu le 20 janvier 1986)
}

\begin{abstract}
RÉSUME
La spectrométrie $X$ et $\gamma$ de faible énergie permet la mesure de faibles quantités de plutonium sans préparation préalable des échantillons. Quelques mesures ont été faites pour préciser les activités et masses limites des échantillons. Si la composition isotopique est connue, les masses de plutonium détectables doivent être de l'ordre du microgramme mais, dans le cas contraire, elles sont plus importantes, de l'ordre du milligramme.
\end{abstract}

\section{ABSTRACT}

The spectrometry for $\mathbf{X}$ - and low-energy $\gamma$ - rays can measure low quantities of plutonium without preliminary preparation. Some measurements have been made to specify the lower limits in activity and mass of samples. If the isotopic composition is known, the detectable plutonium masses should be about one microgram, in the other case, they are more important, about one milligram.

\section{INTRODUCTION}

Le développement de l'industrie nucléaire implique la mise en œuvre de méthodes de plus en plus sensibles, capables de déterminer des quantités de plutonium ou d'uranium présentes parfois à l'état de traces dans des échantillons et, si possible, de connaître la composition isotopique correspondante [1].

A ce jour, plusieurs procédés font appel à la spectrométrie $\gamma$ [8]; la zone d'énergie habituellement analysée s'étend de $100 \mathrm{keV}$ à $800 \mathrm{keV}$ et les échantillons doivent présenter des masses en plutonium de quelques milligrammes au moins. Mais lorsque le plutonium est à l'état de traces, le rayonnement émis dans la zone de 100 à $500 \mathrm{keV}$ n'est plus détectable; c'est alors que l'exploitation des spectres de photons dans le domaine des basses énergies, de 10 à $100 \mathrm{keV}$, peut s'avérer intéressante en raison de probabilités d'émission $X_{L}$ supérieures, de l'ordre de $10^{-2}$.

A l'aide de solutions liquides de déchets réels d'activité croissante, plusieurs mesures ont permis de préciser les activités et masses limites [3] que doivent présenter les échantillons pour permettre une évaluation de la quantité et déterminer la composition isotopique. Le spectromètre considéré fonctionne en mode direct d'analyse, c'est-à-dire qu'il n'est pas équipé de dispositif anticoïncidence pour réduire le rayonnement diffusé.

* Commissariat à l'énergie atomique, ORIS, Laboratoire de métrologie des rayonnements ionisants (LMRI), 91191 Gif-sur-Yvette Cedex. 


\section{RAYONNEMENT X ET $\gamma$ ÉMIS PAR LE PLUTONIUM}

Dans le domaine des basses énergies, le rayonnement photonique émis par les isotopes du plutonium est caractérisé par des raies $X_{L}$, dont la probabilité d'émission est de plusieurs pour cent, et des raies $\gamma$ moins intenses (tableau I). Les raies $\gamma$, d'énergie inférieure à $100 \mathrm{keV}$, émises par ${ }^{241} \mathrm{Pu}$ et ${ }^{242} \mathrm{Pu}$ sont tellement faibles qu'il est pratiquement impossible de les détecter.

\section{TABLEAU ।}

Principales raies $\boldsymbol{X}$ et $\gamma$ utilisées dans le domaine des basses énergies pour les mesures de plutonium $[4,6,9]$

\begin{tabular}{|c|c|c|c|}
\hline $\begin{array}{l}\text { Radio- } \\
\text { nucléide }\end{array}$ & Type de rayonnement & $\begin{array}{l}\text { Probabilité } \\
\text { d'émission } \\
X \text { ou } \gamma\end{array}$ & $\begin{array}{l}\text { Flux } X \text { ou } \gamma \\
\text { par unite } \\
\text { de masse } \\
\left(X \cdot s^{-1} \cdot g^{-1}\right. \\
\left.\text { ou } \gamma \cdot s^{-1} \cdot g^{-1}\right)\end{array}$ \\
\hline${ }^{238} \mathrm{Pu}$ & $\begin{array}{l}\text { Raies } X \\
\quad \text { Total } U X_{L} \\
U X_{L \beta} \text { a d } 5: 16,4 \text { à } 17,5 \mathrm{keV} \\
\text { UX } X_{L \beta} \quad 1,3,5: 17,1 \text { à } 17,5 \mathrm{keV} \\
\text { Raies } \gamma \\
\quad 43,5 \mathrm{keV} \\
152,7 \mathrm{keV}\end{array}$ & $\begin{array}{l}1,15 \pm 0,0510^{-1} \\
5,6 \pm 0,3 \quad 0^{-2} \\
4,9 \pm 0,310^{-2} \\
\\
3,95 \pm 0,0810^{-4} \\
9,38 \pm 0,0910^{-6}\end{array}$ & $\begin{array}{ll}7,3 & 10^{10} \\
3,5 & 10^{10} \\
3,1 & 10^{10} \\
& \\
2,50 & 10^{8} \\
5,94 & 10^{6}\end{array}$ \\
\hline${ }^{239} \mathrm{Pu}$ & 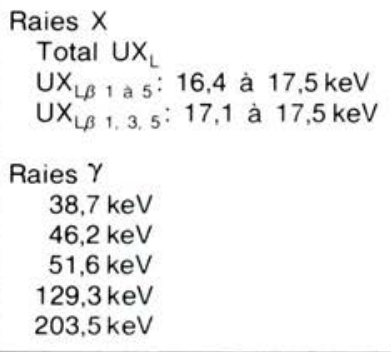 & $\begin{aligned} 5,8 & \pm 0,510^{-2} \\
2,8 & \pm 0,410^{-2} \\
1,9 & \pm 0,310^{-2} \\
& \\
1,02 & \pm 0,0210^{-4} \\
9 & \pm 2 \quad 10^{-6} \\
2,70 & \pm 0,0410^{-4} \\
6,23 & \pm 0,0610^{-5} \\
5,68 & \pm 0,04 \quad 10^{-6}\end{aligned}$ & $\begin{array}{ll}1,33 & 10^{8} \\
6,4 & 10^{7} \\
4,4 & 10^{7} \\
& \\
2,34 & 10^{5} \\
2,1 & 10^{4} \\
6,2 & 10^{5} \\
1,43 & 10^{5} \\
1,30 & 10^{4}\end{array}$ \\
\hline $240 \mathrm{Pu}$ & $\begin{array}{l}\text { Raies } X \\
\quad \text { Total UX } \\
\text { UX } \\
U X_{L \beta} 1 \text { a } 5: 16,4 \text { à } 17,5 \mathrm{keV} \\
\text { UX } 1,3,5: 17,1 \text { à } 17,5 \mathrm{keV} \\
\text { Raies } \gamma \\
\quad 45,2 \mathrm{keV} \\
160,3 \mathrm{keV}\end{array}$ & $\begin{array}{l}1,04 \pm 0,0710^{-1} \\
5,4 \pm 0,4 \quad 10^{-2} \\
4,7 \pm 0,310^{-2} \\
4,35 \pm 0,0910^{-4} \\
4,02 \pm 0,0410^{-6}\end{array}$ & $\begin{array}{ll}8,7 & 10^{8} \\
4,5 & 10^{8} \\
3,9 & 10^{8} \\
& \\
& \\
3,65 & 10^{6} \\
3,37 & 10^{4}\end{array}$ \\
\hline
\end{tabular}


MESURE DES TRANSACTINIDES PAR SPECTROMETRIE

\begin{tabular}{|c|c|c|c|}
\hline $\begin{array}{l}\text { Radio- } \\
\text { nucléide }\end{array}$ & Type de rayonnement & $\begin{array}{l}\text { Probabilité } \\
\text { d'émission } \\
X \text { ou } \gamma\end{array}$ & $\begin{array}{c}\text { Flux } X \text { ou } \gamma \\
\text { par unité } \\
\text { de masse } \\
\left(X . \mathbf{s}^{-1} \cdot \mathrm{g}^{-1}\right. \\
\left.\text { ou } \gamma . \mathrm{s}^{-1} \cdot \mathrm{g}^{-1}\right)\end{array}$ \\
\hline${ }^{241} \mathrm{Pu}-237 \mathrm{U}$ & $\begin{array}{l}\text { Raies } X \\
\text { Total UX } \\
\text { UX } X_{L \beta} 1 \text { à } 5: 16,4 \text { à } 17,5 \mathrm{keV} \\
\text { Raies } \gamma \\
148,6 \mathrm{keV} \\
164,6 \mathrm{keV} \\
208,0 \mathrm{keV}\end{array}$ & $\begin{array}{l}1,20 \pm 0,1010^{-5} \\
6,0 \pm 1,110^{-6} \\
1,88 \pm 0,0310^{-6} \\
4,50 \pm 0,1510^{-7} \\
5,32 \pm 0,1810^{-6}\end{array}$ & $\begin{array}{ll}4,6 & 10^{7} \\
2,3 & 10^{7} \\
& \\
7,2 & 10^{6} \\
1,72 & 10^{6} \\
2,03 & 10^{7}\end{array}$ \\
\hline${ }^{242} \mathrm{Pu}$ & $\begin{array}{l}\text { Raies } X \\
\text { Total } \cup X_{L} \\
\quad U X_{L \beta} 1 \text { a } 5: 16,4 \text { à } 17,5 \mathrm{keV} \\
\text { Raies } \gamma \\
\quad 44,9 \mathrm{keV}\end{array}$ & $\begin{array}{l}\sim 810^{-2} \\
\sim 410^{-2} \\
\sim 410^{-4}\end{array}$ & $\begin{array}{ll}\sim 1,2 & 10^{7} \\
\sim 6 & 10^{6} \\
& \\
\sim 6 & 10^{4}\end{array}$ \\
\hline${ }^{241} \mathrm{Am}$ & $\begin{array}{l}\text { Raies } X \\
\text { Total } N_{P} X_{L} \\
X L_{\beta} \text { i a } 5: 16,8 \text { à } 18,0 \mathrm{keV} \\
X L_{\beta} 1,3,5: 17,5 \text { à } 18,0 \mathrm{keV} \\
\text { Raies } \gamma \\
26,4 \mathrm{keV} \\
59,6 \mathrm{keV} \\
125,3 \mathrm{keV}\end{array}$ & $\begin{array}{l}3,60 \pm 0,0810^{-1} \\
1,80 \pm 0,0610^{-1} \\
1,28 \pm 0,0910^{-1} \\
\\
2,41 \pm 0,0610^{-2} \\
3,60 \pm 0,0310^{-1} \\
4,17 \pm 0,1310^{-5}\end{array}$ & $\begin{array}{ll}4,5 & 10^{10} \\
2,28 & 10^{10} \\
1,62 & 10^{9} \\
& \\
3,05 & 10^{9} \\
4,55 & 10^{10} \\
5,3 & 10^{6}\end{array}$ \\
\hline
\end{tabular}

A partir des mesures faites d'après la raie $X_{L \beta}$ de $17 \mathrm{keV}$, le passage de l'activité d'un échantillon de plutonium à la masse dépend fortement de la proportion relative de ses isotopes. Ainsi, l'isotope principal devient le ${ }^{238} \mathrm{Pu}$ puisque le flux X émis par unité de masse de cet isotope est au moins 100 fois supérieur à celui émis par les autres isotopes. Toute mesure de quantité à partir des raies $X_{L}$ nécessite donc une connaissance préalable de la composition isotopique ou bien sa détermination préalable par spectrométrie $X$ et $\gamma$. En outre, à cause de la présence notable de ${ }^{241} \mathrm{Am}$ au niveau des raies $X_{L}$, une correction d'interférence à $17,8 \mathrm{keV}$ doit être faite d'après les données relevées pour les raies $\gamma$ de $26,4 \mathrm{keV}$ et $59,6 \mathrm{keV}$.

\section{DESCRIPTION DES ESSAIS}

Un détecteur au germanium intrinsèque à structure planaire, de volume $3 \mathrm{~cm}^{3}, \mathrm{a}$ été utilisé ; celui-ci présentait une résolution de $0,24 \mathrm{keV}$ à $6 \mathrm{keV}\left({ }^{55} \mathrm{Fe}\right)$ et de $0,54 \mathrm{keV}$ à $122 \mathrm{keV}\left({ }^{57} \mathrm{Co}\right)$ et il avait été étalonné avec des solutions d'activité connue [5]: ${ }^{57} \mathrm{Co},{ }^{109} \mathrm{Cd},{ }^{133} \mathrm{Ba},{ }^{139} \mathrm{Ce},{ }^{141} \mathrm{Ce},{ }^{241} \mathrm{Am}$. Qu'il s'agisse de solutions étalons ou de solutions de déchets réels, la géométrie adoptée correspondait à celle 
d'un flacon SG 50 [2] rempli de $50 \mathrm{~cm}^{3}$ de solution, placé au contact de la fenêtre d'entrée. Compte tenu de cette géométrie, favorable à la détection, des corrections des pertes de comptage par coïncidence X-X, X- $\gamma$ et $\gamma \gamma$ ont été nécessaires [7] (tableau II). La figure 1 représente la réponse observée en efficacité d'absorption totale.

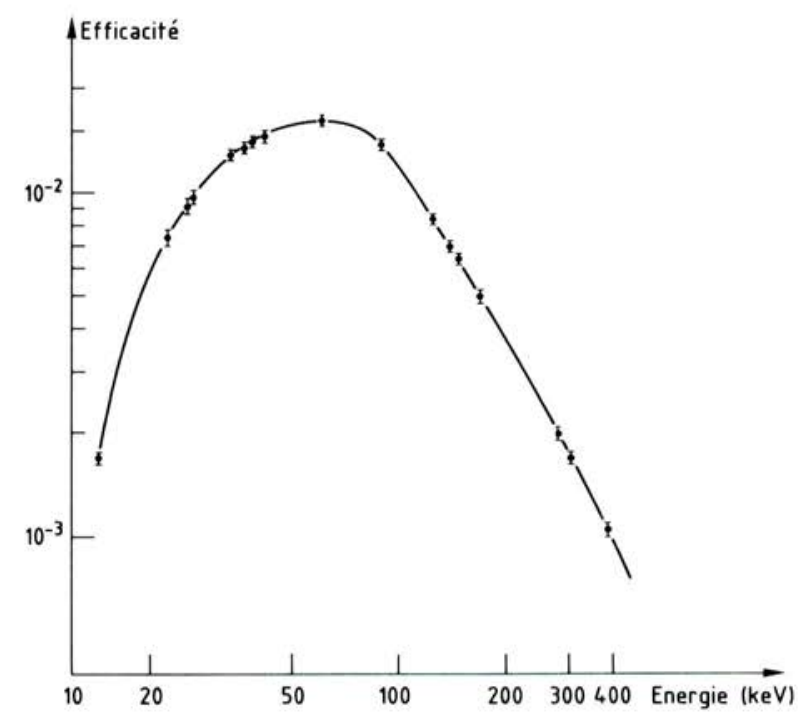

Fig. 1. - Efficacité d'absorption totale GeHP $3 \mathrm{~cm}^{3}$ Echantillon: flacon $50 \mathrm{~cm}^{3}$ au contact.

\section{TABLEAU ॥}

Exemple de correction des coïncidences $X-X, X-\gamma, \gamma \gamma$ pour le cobalt 57

\begin{tabular}{|c|c|c|c|}
\hline Géométrie & \multicolumn{2}{|c|}{ Source ponctuelle } & Flacon de $50 \mathrm{~cm}^{3}$ \\
\hline $\begin{array}{c}\text { Energie } \\
\text { (en keV) }\end{array}$ & à $0,2 \mathrm{~cm}$ & à $5,0 \mathrm{~cm}$ & au contact \\
\hline $6,5 \mathrm{Xk}$ & 1,253 & 1,012 & 1,020 \\
14,4 & 1,210 & 1,010 & 1,018 \\
122,1 & 1,089 & 1,004 & 1,004 \\
136,5 & 0,964 & 0,998 & 0,998 \\
\hline
\end{tabular}




\section{RÉSULTATS}

Le tableau III fait apparaître les valeurs limites à partir desquelles l'activité et la masse de plutonium en fonction de l'activité totale volumique de l'échantillon peuvent être obtenues de l'analyse de la raie $X_{L B}$ de $17 \mathrm{keV}$ ainsi que les valeurs limites concernant le dosage de ${ }^{241} \mathrm{Am}$ après exploitation de la raie de $60 \mathrm{keV}$; la durée de comptage considérée est de $15 \mathrm{~h}$. On entend par activité totale volumique la somme des activités des différents radionucléides présents : émetteurs $\alpha$, produits d'activation, produits de fission - mesurées par spectrométrie $\alpha$ et par spectrométrie $\gamma$. La figure 2 fournit une illustration des résultats précédents.

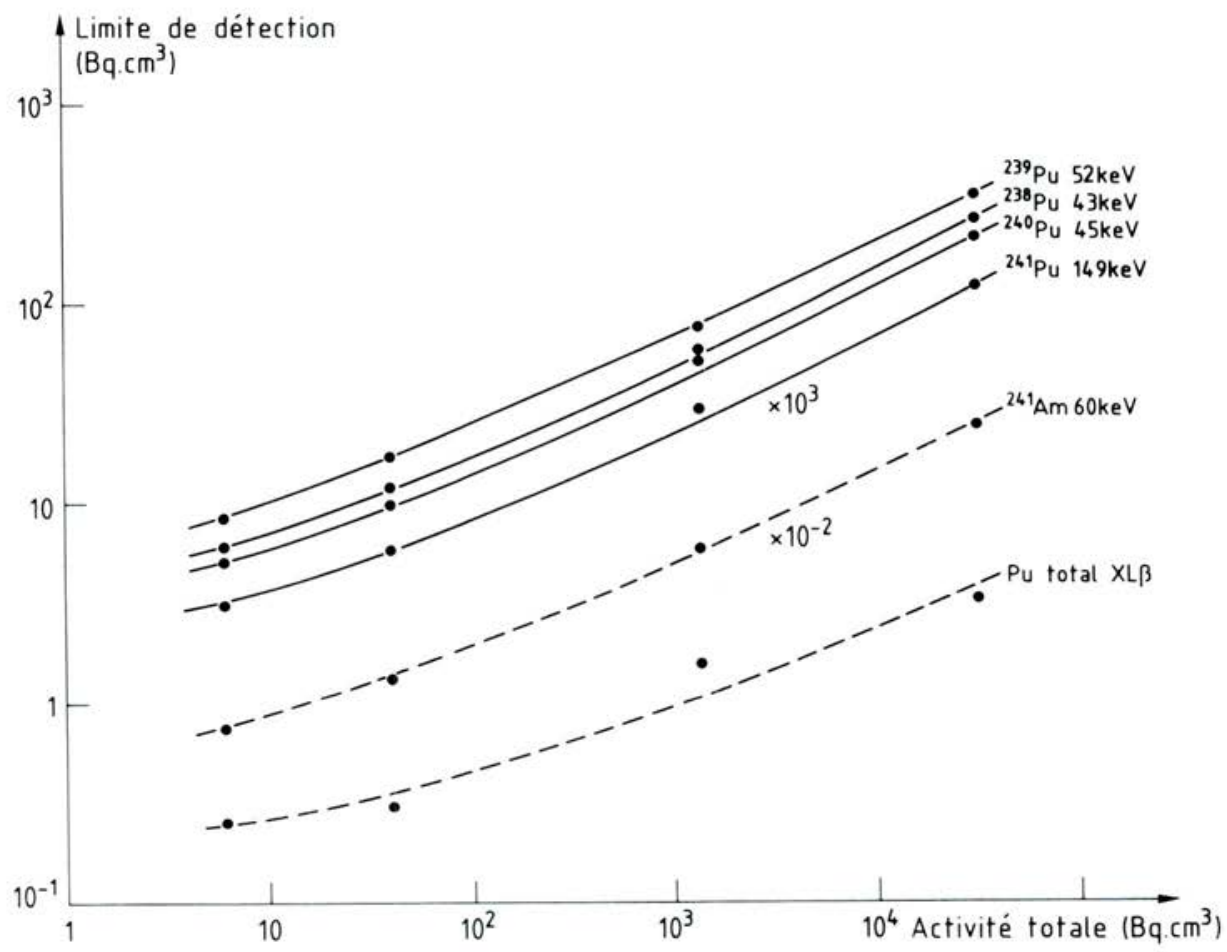

Fig. 2. - Limite de détection en fonction de l'activité totale de l'échantillon pour une mesure de masse $(---)$, une détermination de la composition isotopique $(-)$,

(Détecteur GeHP $3 \mathrm{~cm}^{3}$, volume mesuré: $50 \mathrm{~cm}^{3}$, durée de comptage : $15 \mathrm{~h}$ ). 
TABLEAU III

Limites de détection pour une mesure des quantités de plutonium (Echantillon de $50 \mathrm{~cm}^{3}$. Durée: $15 \mathrm{~h}$ )

\begin{tabular}{|c|c|c|c|c|}
\hline \multirow[b]{2}{*}{ Limites de detection } & \multicolumn{4}{|c|}{ Activité totale de l'échantilion (Bq. $\left.\mathrm{cm}^{-3}\right)$} \\
\hline & 6 & 40 & 1400 & 30000 \\
\hline $\begin{array}{l}\text { en Bq.cm-3 } \\
\text { Pu total (UL } \beta)^{\star} \\
{ }^{241} \mathrm{Am}(60 \mathrm{keV})\end{array}$ & $\begin{array}{l}0,25 \\
0,007\end{array}$ & $\begin{array}{l}0,30 \\
0,013\end{array}$ & $\begin{array}{l}1,6 \\
0,060\end{array}$ & $\begin{array}{l}3,4 \\
0,26\end{array}$ \\
\hline 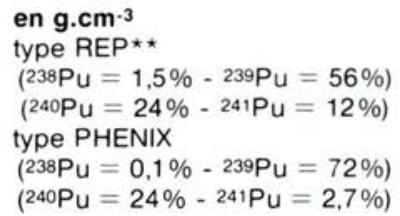 & $\begin{array}{l}1,8 \times 10^{-11} \\
7 \times 10^{-11}\end{array}$ & $\begin{array}{l}2,1 \times 10^{-11} \\
8 \times 10^{-11}\end{array}$ & $\begin{array}{l}1,1 \times 10^{-10} \\
4 \times 10^{-10}\end{array}$ & $\begin{array}{l}2.4 \times 10^{-10} \\
9 \times 10^{-10}\end{array}$ \\
\hline
\end{tabular}

* Tous les isotopes du Pu.

$\star \star$ REP : réacteur à eau sous pression.

Par rapport aux mesures $\alpha$ faites en chambre à grille ou avec un détecteur semiconducteur où les valeurs usuelles sont inférieures à $0,01 \mathrm{~Bq} \cdot \mathrm{cm}^{-3}$, les valeurs limites obtenues en spectrométrie de photons de faible énergie sont nettement plus élevées, de l'ordre du Bq. $\mathrm{cm}^{-3}$. Cependant, cette dernière technique permet de s'affranchir des difficultés rencontrées en spectrométrie $\alpha$ liées à la préparation des échantillons: séparation chimique, évaporation, concentration, électrodéposition, etc. Compte tenu du fait que les valeurs limites observées dans la région des raies $X_{L}$ sont d'autant plus importantes que le fond dans cette région l'est également, ce bruit étant imputable à la détection des raies d'énergies plus élevées et en particulier à la détection des produits d'activation et des produits de fission, il serait possible d'optimiser les conditions expérimentales; de meilleures limites seraient obtenues en diminuant l'épaisseur du détecteur, en augmentant sa surface, en diminuant aussi l'épaisseur de l'échantillon.

Le passage de l'activité à la masse de plutonium oblige de connaître la composition isotopique, c'est-à-dire les rapports $238 \mathrm{Pu} / \mathrm{Pu},{ }^{239} \mathrm{Pu} / \mathrm{Pu},{ }^{240} \mathrm{Pu} / \mathrm{Pu}$, ${ }^{241} \mathrm{Pu} / \mathrm{Pu},{ }^{242} \mathrm{Pu} / \mathrm{Pu}$. S'il s'agit d'une unité traitant le même type de combustibles nucléaires, la composition isotopique peut être admise a priori; par contre, si les combustibles traités sont divers ou bien si l'expérimentateur ne dispose pas d'informations sur l'échantillon mesuré, il faut faire une détermination de la composition isotopique avant toute mesure de masse.

Dans le domaine des basses énergies, les rapports $238 \mathrm{Pu} / 239 \mathrm{Pu}$, ${ }^{240} \mathrm{Pu} / 239 \mathrm{Pu},{ }^{241} \mathrm{Pu} / 239 \mathrm{Pu},{ }^{241} \mathrm{Am} / 239 \mathrm{Pu}$ sont obtenus à partir de l'analyse des pics suivants: 43,5 et $51,6 \mathrm{keV}-44,9$ et $51,6 \mathrm{keV}-148,6$ et $129,3 \mathrm{keV}-59,6$ et 
$51,6 \mathrm{keV}$; le rapport ${ }^{242} \mathrm{Pu} / 239 \mathrm{Pu}$ est déduit d'une corrélation faisant intervenir les rapports précédents. La réponse en efficacité d'absorption totale est établie de manière relative en utilisant les informations relevées par les pics $38,7 \mathrm{keV}$ $51,6 \mathrm{keV}-129,3 \mathrm{keV}-203,0 \mathrm{keV}$ - du $239 \mathrm{Pu}$.

Les valeurs limites permettant un dosage des isotopes du plutonium, donc une détermination de la composition isotopique, sont présentées dans le tableau IV. En raison de l'absence de raies $\gamma$ intenses dans la région de $10 \mathrm{keV}$ à $100 \mathrm{keV},{ }^{241} \mathrm{Pu}$ ne peut être dosé qu'à partir des raies $148,6 \mathrm{keV}$ et $164,5 \mathrm{keV}$. Les valeurs limites à adopter pour le dosage des composantes du plutonium seront égales aux valeurs limites du ${ }^{24} \mathrm{Pu}$ si la proportion en masse de cet isotope est importante.

TABLEAU IV

Limites de détection pour une détermination de la composition isotopique (échantillon de $50 \mathrm{~cm}^{3}$, durée $15 \mathrm{~h}$ )

\begin{tabular}{|c|c|c|c|c|}
\hline \multirow{2}{*}{ Limites de détection } & \multicolumn{4}{|c|}{ Activité totale de l'échantillon $\left(\mathrm{Bq} . \mathrm{cm}^{-3}\right)$} \\
\hline & 6 & 40 & 1400 & 30000 \\
\hline en Bq. $\mathrm{cm}^{-3}$ & & & & \\
\hline${ }^{238} \mathrm{Pu}(43 \mathrm{keV})$ & 6,0 & 11 & 59 & 270 \\
\hline 2399 Pu (52 keV) & 8,6 & 17 & 77 & 330 \\
\hline $240 \mathrm{Pu}(45 \mathrm{keV})$ & 5,4 & 10 & 52 & 210 \\
\hline${ }^{241} \mathrm{Pu}(149 \mathrm{keV})$ & $3,2 \times 10^{3}$ & $6,0 \times 10^{3}$ & $3,0 \times 10^{4}$ & $1,3 \times 10^{5}$ \\
\hline en g.cm ${ }^{-3}-241 \mathrm{Pu}(149 \mathrm{keV})$ & & & & \\
\hline${ }^{241} \mathrm{Pu}$ & $8 \times 10 \cdot 10$ & $1,6 \times 10^{-9}$ & $8 \times 10^{-9}$ & $3,4 \times 10^{-8}$ \\
\hline Pu total - REP & $7 \times 10^{-9}$ & $1,3 \times 10^{-8}$ & $6 \times 10^{-8}$ & $3 \times 10^{-7}$ \\
\hline Pu total - PHENIX & $3 \times 10^{-8}$ & $6 \times 10^{-8}$ & $3 \times 10^{-7}$ & $1,2 \times 10^{-6}$ \\
\hline
\end{tabular}

\section{CONCLUSION}

Actuellement, la spectrométrie $X$ et $\gamma$ de faible énergie ne permet pas de mesurer du plutonium à l'état de traces comme cela est le cas avec la spectrométrie $\alpha$; cependant, elle présente l'avantage de permettre une mesure directe des prélèvements sans aucune préparation préalable. En admettant une composition isotopique connue, les masses qui peuvent être détectées sont de l'ordre du microgramme pour une durée de comptage d'une heure. Pour une détermination de la composition isotopique, les quantités doivent être plus importantes, de l'ordre du milligramme. 


\section{RÉFÉRENCES BIBLIOGRAPHIQUES}

[1] EUROPEAN SAFEGUARDS RESEARCH AND DEVELOPMENT ASSOCIATION (ESARDA). 7. Annual symposium on safeguards and nuclear material management, Liege, May 1985. Ispra: ESARDA, 1985.

[2] CEA , GROUPE DE TRAVAIL GTR5. Normalisation des flacons utilisés en spectrométrie $\gamma$. Rapport CEA-R-5145, 1982.

[3] CEA, GROUPE DE TRAVAIL SPECTROMETRIE $\gamma$. Limite de détection au rayonnement $\gamma$ des spectromètres équipés de dètecteur à semi-conducteur. Rapport CEA-R-4919, 1978.

[4] GUNNINK R., EVANS J.E., PRINDLE A.L. Reevaluation of the $\gamma$-ray energies and absolute branching intensities of $237 \mathrm{U}, 238-239-240-241 \mathrm{Pu}$, and ${ }^{241} \mathrm{Am}$. Rapport UCRL-52139, 1976.

[5] LABORATOIRE DE METROLOGIE DES RAYONNEMENTSIONISANTS (LMRI), CEA-ORIS. Etalons de radioactivité. Gif-sur-Yvette: CEA-LMRI, 1985.

[6] LAGOUTINE F., COURSOL N. Table de radionucléides du Laboratoire de métrologie des rayonnements ionisants. Gif-sur-Yvette: CEA-LMRI, 1983-1984.

[7] MOREL J., CHAUVENET B., KADACHI A. Coincidence - summing corrections in gammaray spectrometry for normalized geometries. Int. J. Appl. Radiat. Isot., 1983, 34 (8), 11151122.

[8] MOREL J., VALLEE M., CHAUVENET B. Composition isotopique du plutonium par spectrométrie gamma ; programme d'analyse automatique. Note CEA-N-2303, 1982.

[9] SHERMAN I.S., STRAUSS M.G., PEHL R.H. Measurement of trace radionuclides in soil by $L_{x}$ ray spectrometry. IEEE Trans. Nucl. Sci., 1980, NS 27 (1) 695-703. 